\title{
Josephson oscillation and induced collapse in an attractive Bose-Einstein condensate
}

\author{
Sadhan K. Adhikari \\ Instituto de Física Teórica, Universidade Estadual Paulista, 01.405-900 São Paulo, São Paulo, Brazil
}

(Received 26 October 2004; published 28 July 2005)

\begin{abstract}
Using the axially symmetric time-dependent Gross-Pitaevskii equation we study the Josephson oscillation of an attractive Bose-Einstein condensate (BEC) in a one-dimensional periodic optical-lattice potential. We find that the Josephson frequency is virtually independent of the number of atoms in the BEC and of the interatomic interaction (attractive or repulsive). We study the dependence of the Josephson frequency on the laser wave length and the strength of the optical-lattice potential. For a fixed laser wave length $(795 \mathrm{~nm})$, the Josephson frequency decreases with increasing strength as found in the experiment of Cataliotti et al. [Science 293, 843 (2001)]. For a fixed strength, the Josephson frequency remains essentially unchanged for a reasonable variation of laser wave length around $800 \mathrm{~nm}$. However, the Josephson oscillation is disrupted with the increase of laser wave length beyond $2000 \mathrm{~nm}$ leading to a collapse of a sufficiently attractive BEC. These features of a Josephson oscillation can be tested experimentally with present setups.
\end{abstract}

DOI: 10.1103/PhysRevA.72.013619

PACS number(s): 03.75.Lm, 03.75.Kk

\section{INTRODUCTION}

The observation of an oscillating Josephson current across a one-dimensional array of potential wells, usually generated by a standing-wave laser field and commonly known as an optical-lattice potential, in a trapped cigar-shaped BoseEinstein condensate (BEC) by Cataliotti et al. [1] was a manifestation of this phenomenon in trapped neutral bosons. Until then the Josephson effect was confirmed in superconductors with charged electrons and in liquid helium [2].

In the experiment of Cataliotti et al. [1] a Josephson oscillation was initiated in a repulsive ${ }^{87} \mathrm{Rb}$ BEC formed in a one-dimensional optical-lattice plus axially symmetric harmonic traps by suddenly displacing the harmonic trap along the axial direction. They investigated the evolution of the Josephson frequency with the strength of the optical-lattice potential and found that, for a fixed laser wave length, the Josephson frequency reduces with the increase of the strength.

There have been previous theoretical studies of the Josephson oscillation to explain different features of the experiment of Cataliotti et al. [1] using the numerical solution of the time-dependent mean-field Gross-Pitaevskii (GP) equation [3]. One-dimensional $[1,4,5]$ and three-dimensional [6] models based on the GP equation have previously been used to study different aspects of Josephson oscillation. There have been other theoretical studies of the Josephson oscillation in a trapped BEC [7-9] using different approaches and under different conditions.

In this paper we address several interesting features of the Josephson oscillation in a trapped BEC as in the experiment of Cataliotti et al. [1]. How does the Josephson frequency evolve with the number of atoms and atomic interaction (attractive or repulsive) of the condensate? How does the Josephson frequency depend on the intensity (strength) and wavelength of the standing-wave laser beam used to create the periodic optical-lattice potential? What are the limits on the optical-lattice potential parameters for allowing a Josephson oscillation? Is it possible to have a collapse in an attractive BEC induced by a Josephson oscillation?

In the actual experiment [1] and a previous threedimensional simulation [6] the extraction of the Josephson frequency was rather indirect. The BEC in the optical-lattice potential was allowed to expand and form an interference pattern of two bright pieces moving in opposite directions from a central piece after a certain interval of time. The position of the original BEC executing a Josephson oscillation in the periodic optical-lattice potential was predicted from the position of the observed interference pattern. In this indirect fashion the Josephson oscillation was identified and its frequency extracted. Although, because of the very small size of the BEC, this seems to be the only means for experimental observation, we employ a direct approach for the study of a Josephson oscillation in the present numerical simulation, which is more accurate and easier to implement than the indirect approach employed in Refs. $[1,6]$. In present simulation we directly follow the oscillating condensate without resorting to an expansion.

There have been experimental [10] and theoretical [11] studies of the collapse of a ${ }^{85} \mathrm{Rb}$ BEC after suddenly turning the atomic interaction from repulsive to attractive by manipulating an external magnetic field near a Feshbach resonance [12]. There has also been a study of induced collapse of an attractive BEC trapped by a system of laser beams in the presence of small fluctuations of the laser intensity [13]. In this paper, to the best of our knowledge, we study for the possibility of collapse of an attractive BEC induced by a Josephson oscillation. In view of the routine experiments $[14,15]$ on BECs formed in optical-lattice potentials, it should be possible to verify the predictions of the present study on the Josephson oscillation of an attractive BEC.

In Sec. II we present the mean-field model based on the axially symmetric time-dependent nonlinear GP equation. In Sec. III we present the numerical results and finally, in Sec. IV we present a summary of our study. 


\section{MEAN-FIELD MODEL}

The time-dependent BEC wave function $\Psi(\mathbf{r} ; t)$ at position $\mathbf{r}$ and time $t$ is described by the following mean-field nonlinear GP equation [3]

$$
\left[-i \hbar \frac{\partial}{\partial t}-\frac{\hbar^{2} \nabla^{2}}{2 m}+V(\mathbf{r})+g N|\Psi(\mathbf{r} ; t)|^{2}\right] \Psi(\mathbf{r} ; t)=0,
$$

where $m$ is the mass and $N$ the number of atoms in the condensate, $g=4 \pi \hbar^{2} a / m$ the strength of interatomic interaction, with $a$ as the atomic scattering length. In the presence of the combined axially symmetric and periodic opticallattice potentials $V(\mathbf{r})=\frac{1}{2} m \omega^{2}\left(\rho^{2}+\nu^{2} y^{2}\right)+V_{\text {opt }}$ where $\omega$ is the angular frequency of the harmonic potential in the radial direction $\rho, \nu \omega$ that in the axial direction $z$, with $\nu$ as the aspect ratio, and $V_{\mathrm{opt}}=s E_{R} \cos ^{2}\left(k_{L} z\right)$ is the optical-lattice potential created with the standing-wave laser field of wavelength $\lambda$ $=795 \mathrm{~nm}$, as in the experiment of Ref. [1], with $E_{R}$ $=\hbar^{2} k_{L}^{2} /(2 m), k_{L}=2 \pi / \lambda$, and $s(<12)$ the strength. The normalization condition is $\int d \mathbf{r}|\Psi(\mathbf{r} ; t)|^{2}=1$.

In the axially symmetric configuration, the wave function can be written as $\Psi(\mathbf{r}, t)=\psi(\rho, z, t)$, where $0 \leqslant \rho<\infty$ is the radial variable and $-\infty<z<\infty$ is the axial variable. Now transforming to dimensionless variables $\hat{\rho}=\sqrt{2} \rho / l, \hat{z}=\sqrt{2} z / l$, $\tau=t \omega, l \equiv \sqrt{\hbar /(m \omega)}$, and $\varphi(\hat{\rho}, \hat{z} ; \tau) \equiv \hat{\rho} \sqrt{l^{3} / \sqrt{8}} \psi(\rho, z ; t)$, Eq. (1) becomes [18]

$$
\begin{aligned}
& {\left[-i \frac{\partial}{\partial \tau}-\frac{\partial^{2}}{\partial \hat{\rho}^{2}}+\frac{1}{\hat{\rho}} \frac{\partial}{\partial \hat{\rho}}-\frac{\partial^{2}}{\partial \hat{z}^{2}}+\frac{1}{4}\left(\hat{\rho}^{2}+\nu^{2} \hat{z}^{2}\right)-\frac{1}{\hat{\rho}^{2}}\right.} \\
& \left.\quad+s \frac{E_{R}}{\hbar \omega} \cos ^{2}\left(k_{L} z\right)+8 \sqrt{2} \pi n\left|\frac{\varphi(\hat{\rho}, \hat{z} ; \tau)}{\hat{\rho}}\right|^{2}\right] \varphi(\hat{\rho}, \hat{z} ; \tau)=0,
\end{aligned}
$$

where the nonlinearity parameter $n=N a / l$. In terms of the one-dimensional probability $P(z, t) \equiv 2 \pi \int_{0}^{\infty} d \hat{\rho}|\varphi(\hat{\rho}, \hat{z}, \tau)|^{2} / \hat{\rho}$, the normalization of the wave function is given by $\int_{-\infty}^{\infty} d \hat{z} P(z, t)=1$.

The experiment of Cataliotti et al. [1] was performed with repulsive ${ }^{87} \mathrm{Rb}$ atoms in the hyperfine state $F=1, m_{F}=-1$. The axial and radial trap frequencies were $\nu \omega=2 \pi \times 9 \mathrm{~Hz}$ and $\omega=2 \pi \times 92 \mathrm{~Hz}$, respectively, with $\nu=9 / 92$. In the present simulation we consider attractive ${ }^{85} \mathrm{Rb}$ atoms in the hyperfine state $F=2, m_{F}=-2$ with mass quite close to ${ }^{87} \mathrm{Rb}$ atoms. The triplet scattering length $a_{T}$ for the ${ }^{85} \mathrm{Rb}-{ }^{85} \mathrm{Rb}$ system is negative corresponding to attraction: $-500 a_{0}<a_{T}<$ $-300 a_{0}$ with $a_{0}=0.5292 \AA$ the Bohr radius [16]. Also, in case of ${ }^{85} \mathrm{Rb}$ the atomic interaction can be modified using a Feshbach resonance [12]. So the ${ }^{85} \mathrm{Rb}^{-}{ }^{85} \mathrm{Rb}$ system is the most suitable for studying the dependence of a Josephson oscillation on the atomic interaction.

For ${ }^{85} \mathrm{Rb}$ the harmonic-oscillator length $l=\sqrt{\hbar /(m \omega)}$ $=1.14 \mu \mathrm{m}$ and the present dimensionless length unit corresponds to $l / \sqrt{2}=0.8 \mu \mathrm{m}$. The present dimensionless time unit corresponds to $\omega^{-1}=1 /(2 \pi \times 92) s=1.73 \mathrm{~ms}$. Although we perform the calculation in dimensionless units using Eq. (2), we present the results in actual physical units using these conversion factors consistent with ${ }^{85} \mathrm{Rb}$ atoms. In terms of the dimensionless laser wave length $\lambda_{0}=\sqrt{2} \lambda / l \simeq 1$, the di- mensionless standing-wave energy parameter $E_{R} /(\hbar \omega)$ $=4 \pi^{2} / \lambda_{0}^{2}$. Hence, in dimensionless unit the periodic opticallattice potential of Eq. (2) is

$$
\frac{V_{\mathrm{opt}}}{\hbar \omega} \equiv s \frac{E_{R}}{\hbar \omega} \cos ^{2}\left(k_{L} z\right)=s \frac{4 \pi^{2}}{\alpha^{2} \lambda_{0}^{2}}\left[\cos ^{2}\left(\frac{2 \pi}{\alpha \lambda_{0}} \hat{z}\right)\right],
$$

where we have introduced a parameter $\alpha$ to manipulate the laser wave length. Normally, $\alpha=1$ corresponds to the experimental situation: $\lambda=795 \mathrm{~nm}$. A general value for $\alpha$ simulates a laser wave length of $\alpha \lambda$.

We solve Eq. (2) numerically using a split-step timeiteration method with the Crank-Nicholson discretization scheme described recently [17]. We discretize the GP equation typically with time step 0.001 and space step 0.1 spanning $\rho$ from 0 to $7 \mu \mathrm{m}$ and $z$ from -70 to $70 \mu \mathrm{m}$, although, sometimes we used smaller steps for obtaining convergence. The time iteration is started with the known harmonic oscillator solution of Eq. (2) for $n=0: \quad \varphi(\hat{\rho}, \hat{z})$ $=\left[\nu /\left(8 \pi^{3}\right)\right]^{1 / 4} \hat{\rho} e^{-\left(\hat{\rho}^{2}+\nu \hat{z}^{2}\right) / 4}$ with chemical potential $\bar{\mu}=(1$ $+\nu / 2$ ) [18]. For a typical cigar-shaped condensate with $\nu$ $\simeq 0.1[1] \bar{\mu} \simeq 1$ is much smaller than the typical depth of the optical-lattice potential wells $E_{R} /(\hbar \omega)=4 \pi^{2} / \lambda_{0}^{2} \simeq 40$ so that $\bar{\mu} \ll E_{R} /(\hbar \omega)$ and the passage of condensate atoms from one well to other can only proceed through quantum tunneling. The nonlinearity $n$ as well as the optical-lattice potential parameter $s$ are slowly increased by equal amounts in $10000 n$ steps of time iteration until the desired value of nonlinearity and optical-lattice potentials are attained. Then, without changing any parameter, the solution so obtained is iterated 50000 times so that a stable solution is obtained independent of the initial input and time and space steps. The solution then corresponds to the bound BEC under the joint action of the harmonic and periodic optical-lattice potentials.

\section{NUMERICAL RESULTS}

First we consider an attractive BEC formed in the combined harmonic and periodic optical-lattice potentials for a specific attractive (negative) nonlinearity $n$. Such a BEC is stable for $|n|$ less than a critical value depending on the parameters of the optical-lattice potential [19]. We study the formation of a BEC in the combined harmonic and opticallattice potentials for $\alpha=1$ in Eq. (3) for a range of values of $s$. Depending on the value of $\alpha$, the BEC could be unstable for $|n|>0.4$ [19] and for $\alpha=1$ we consider $n=-0.3$. However, for a larger $\alpha$, the BEC is stable for a larger value of $|n|$ [19] (see, for example, the stable condensate studied in Fig. 5 for $n=-0.5$ in the following for $\alpha=3$ ). In Fig. 1 the plot of $|\psi(\rho, z)| \operatorname{vs} \rho$ and $z$ illustrates the profile of the BEC bound state for $n=-0.3$ and $s=4$. The BEC is in the form of narrow slices with the optical-lattice barriers separating the slices. The large number of maxima and minima due to the opticallattice potential is not clearly visible in this plot. The maxima and minima in the axial direction are clear in the plot of the one-dimensional probability $P(z) \mathrm{vs} z$ in the off-set of Fig. 1 for $4 \mu \mathrm{m}>z>-4 \mu \mathrm{m}$. In this interval of $z$, there are 16 wells of the optical-lattice potential and as many maxima and minima in $P(y, t)$. 


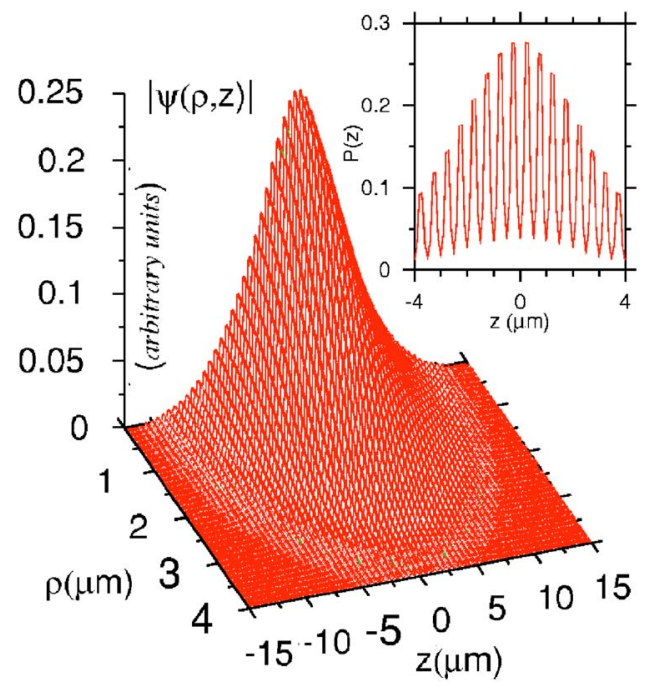

FIG. 1. (Color online) The profile of the BEC wave function $|\psi(\rho, z)|$ formed in the joint optical-lattice and harmonic potentials for $n=-0.3, s=4$, and $\alpha=1$. In the off-set the one-dimensional probability $P(z)$ of this wave function vs $z$ is plotted for $4 \mu \mathrm{m}>z>$ $-4 \mu \mathrm{m}$ which clearly exhibits the maxima and minima of the condensate wave function along the axial direction.

Next we consider an oscillating BEC in the combined harmonic and periodic optical-lattice potentials. If we suddenly displace the harmonic trap along the lattice axis by a small distance after the formation of the BEC in the combined potentials, the condensate will acquire a potential energy, be out of equilibrium and start to oscillate. As the height of the potential-well barriers of the optical-lattice potential is much larger than the energy of the system, the atoms in the condensate will move by tunneling through the potential barriers. This fluctuating transfer of atoms across the potential barriers is due to Josephson effect in a neutral quantum liquid.

With the attractive BEC of Fig. 1 we next study its Josephson oscillation when the harmonic potential is suddenly displaced along the axial direction by $16 \mu \mathrm{m}$. The BEC now acquires an added potential energy which it can use to execute a Josephson oscillation along the axial direction. The Josephson oscillation is best studied numerically from the contour plot of the one-dimensional probability $P(z, t) \mathrm{vs} z$ and $t$ exhibited in Fig. 2. These plots clearly show the central position of the BEC along the axial $z$ direction. In the present simulation we take $\alpha=1$ and different values of the opticallattice strength $s$. These contour plots are very useful to find the Josephson frequencies. From Fig. 2 the periods of the Josephson oscillation are easily read off and the frequencies calculated for different optical-lattice strengths $s$.

In Fig. 3 we plot the Josephson frequencies versus optical-lattice strength $s$ from different studies for $\alpha=1$. Specifically, in addition to the present calculation with attractive atoms we also show (1) the experimental frequencies and corresponding tight-binding calculations for repulsive atoms of Cataliotti et al. [1], (2) the three-dimensional simulation in the repulsive case from Ref. [6], and (3) the hydrodynamical calculation with repulsive atoms from Ref. [7].

The most interesting conclusion from Fig. 3 is that the present frequencies of three-dimensional simulation for an attractive BEC are practically the same as the frequencies of the repulsive BEC of Ref. [6]. Hence, the Josephson frequencies are independent of, or at best weakly dependent on, the number of atoms in the condensate and nature of interaction (repulsive or attractive). For $s=0$ the optical-lattice potential is absent and the condensate executes free oscillation with the frequency of the axial potential $(9 \mathrm{~Hz})$ and the threedimensional calculations by this author converges to this value in the $s \rightarrow 0$ limit for both attractive and repulsive interactions.

However, there are some discrepancies between the present three-dimensional calculation and the tight-binding calculation of Ref. [1] as well as between the present calculation and the hydrodynamical calculation of Ref. [7] for small values of optical-lattice strength $s$. The Josephson frequencies of Refs. [1,7] do not seem to lead to the same result $(9 \mathrm{~Hz})$ as the present calculation in the low $s$ limit. The reason for this could be the possible use of a slightly different axial trap frequency in these studies. For large values of optical-lattice strength $s$, there are no experimental results. In this domain, the present frequencies agree well with the hydrodynamical calculation of Ref. [7]. But both these theoretical results differ from the tight-binding calculation of Ref. [1]. The reason for this could be the use of a variational Gaussian ansatz in Ref. [1].

Now we study the effect of the variation of laser wave length on the Josephson frequency for a fixed strength $s$ of the optical-lattice potential. To implement it we vary the parameter $\alpha$ of the optical-lattice potential (3), which effectively varies the wave length of the laser. The Josephson frequency is found to be weakly dependent on laser wave length for $1600 \mathrm{~nm}>\lambda>600 \mathrm{~nm}$ leading to frequencies $7.1 \pm 0.3 \mathrm{~Hz}$. If the Josephson frequency was solely determined by the effective strength of the optical-lattice potential $\left(4 \pi^{2} / \lambda_{0}^{2}\right) \times\left(s / \alpha^{2}\right)$ of Eq. (3), then from Fig. 3 a much larger variation of frequency would be expected for a variation of $\lambda$ in the range 600-1600 $\mathrm{nm}$. The weak dependence of Josephson frequency on $\alpha$ suggests that in addition to the strength of the optical-lattice potential, the cosine term in Eq. (3) also plays a decisive role in determining the Josephson frequency.

Next, we consider the effect of increasing the laser wave length beyond $\lambda=2000 \mathrm{~nm}$. The condensate in the periodic optical-lattice potential is in the form of narrow slices with the optical-lattice barriers separating the slices (see Fig. 1 and related description). These slices of the BEC have to tunnel through the optical-lattice barriers during a Josephson oscillation. The increase in laser wavelength corresponds to an increase in the widths of the slices of the BEC and optical-lattice barriers and to execute a Josephson oscillation, larger pieces of condensates have to tunnel freely through wider optical-lattice barriers.

With the increase of the laser wave length beyond a critical value, the larger pieces of condensates cannot freely tunnel through wider barriers and there is actually a disruption of Josephson oscillation. This is illustrated in Fig. 4 through a contour plot of one-dimensional probability density $P(z, t)$ with $\alpha=3$ and 4 when the harmonic potential is suddenly displaced along the axial direction through $16 \mu \mathrm{m}$ at $t=0$ for $s=4$ for a small nonlinearity $n=-0.1$. The clean Josephson oscillation tracks seen in Fig. 2 now disappear with the in- 

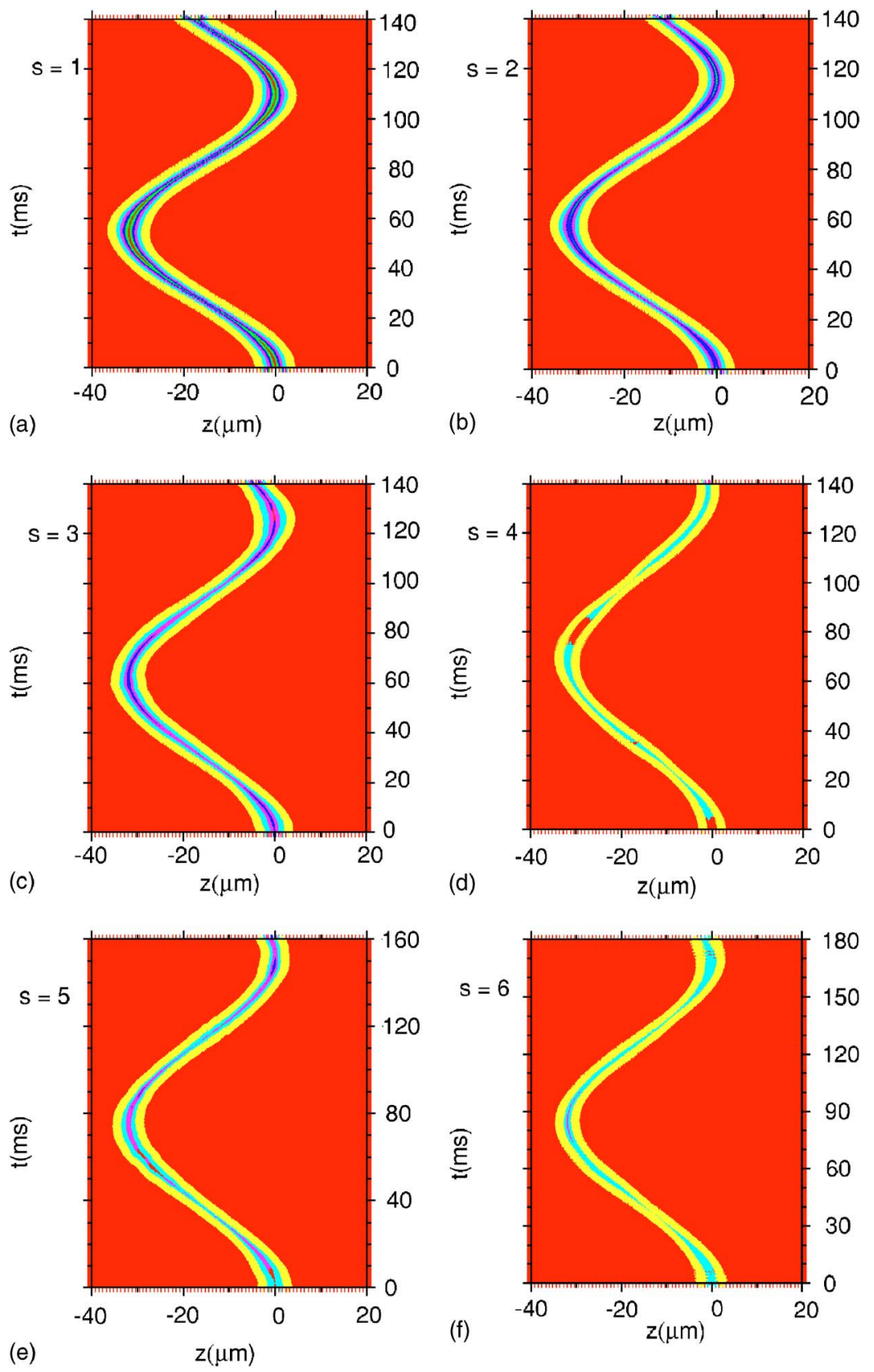

FIG. 2. (Color online) Contour plot of the one-dimensional probability density $P(z, t)$ of the attractive BEC with $\alpha=1$ and $n=-0.3$ executing a Josephson oscillation when the harmonic trap is suddenly displaced along the axial direction through a distance $16 \mu \mathrm{m}$ at $t=0$ for opticallattice strength $s=$ (a) 1, (b) 2, (c) 3, (d) 4, (e) 5, and (f) 6 . The period of the Josephson oscillation can be obtained from these plots. crease of laser wave length as can be seen in Figs. 4(a) and 4(b) where we show the contour of the one-dimensional probability density $P(z, t)$ for $\alpha=3$ and 4. From Figs. 2(d), 4(a), and 4(b) we see that for $s=4$ there is a clean Josephson oscillation track for $\alpha=1$. The same is true for $\alpha=2$. However, with an increase of $\alpha$ to 3 the trail left by the condensate is not so clean showing the beginning of breakdown of the Josephson oscillation in Fig. 4(a). There is a complete breakdown (absence) of the periodic Josephson oscillation in Fig. 4(b) for $\alpha=4$.

There has been a different account of the breakdown of the Josephson oscillation in a neutral quantum fluid in Ref. [20] when the harmonic potential was displaced by a very large distance $(>130 \mu \mathrm{m})$ along the periodic optical-lattice potential. It was suggested that the breakdown of the Josephson oscillation in that study was due to a loss of phase co- herence in the condensate due to a classical transition from a superfluid to an "insulator" resulting in a modulational instability. Other mechanisms for the loss of phase coherence in a condensate have also been studied [21]. The loss of phase coherence considered in all these investigations [20,21] originated from a classical superfluid to insulator transition, different from a quantum transition of a superfluid to a Mott insulator observed in Ref. [14].

From Fig. 3 we find that for a fixed laser wave length, the Josephson frequency reduces with increasing optical-lattice strength. From Fig. 4 we find that for a fixed optical-lattice strength with an increase in the laser wavelength the Josephson oscillation is strongly attenuated. As the Josephson oscillation takes place by quantum tunneling of the atomic superfluid through the optical-lattice barriers, this oscillation is bound to be reduced as the height and width of the optical- 


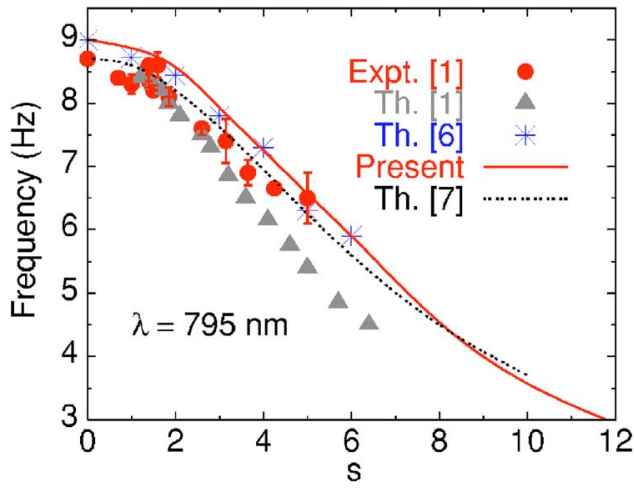

FIG. 3. (Color online) The frequency of the atomic current in the array of Josephson junctions as a function of optical-lattice strength $s$ : - with error bar-experiment of Cataliotti et al. (see Ref. [1]) with repulsive ${ }^{87} \mathrm{Rb}$ atoms; $\triangle$-tight binding calculation taken from Ref. [1]; *- simulation with repulsive ${ }^{87} \mathrm{Rb}$ atoms taken from Ref. [6]; full line-present calculation with attractive atoms; dashed line-hydrodynamical calculation with repulsive atoms taken from Ref. [7].

lattice barriers are increased. The reduction of the Josephson oscillation with the increase of the parameter $s$ results in a reduction of the Josephson frequency in Fig. 3. A breakdown of the Josephson oscillation with increasing $\alpha$ is found in Fig. 4.

Finally, we investigate if the above disruption of the Josephson oscillation for a large $\alpha$ could provoke a collapse in an attractive BEC. We find in our numerical study that in the cases where there is a clean Josephson oscillation there is no collapse in an attractive condensate. However, for large $\alpha$, when there is a disruption of the Josephson oscillation, e.g., as in Fig. 4, one could have a collapse in an attractive condensate with sufficiently strong nonlinearity.

To demonstrate the above claim we performed a simulation of the Josephson oscillation using the parameters of Fig. 2 for attractive nonlinearity close to the critical nonlinearity for collapse [19]. In the actual conditions of Fig. 2, the Josephson oscillation is always perfect and there is no collapse in the condensate provoked by this oscillation. However, the scenario changes as the parameter $\alpha$ is increased for a large nonlinearity $|n|$. To illustrate this we performed calculation with optical-lattice strength $s=4, \alpha=3$, and nonlinearity $n=$ -0.5 . The stationary BEC wave function in this case is localized and stable as shown in Fig. 5(a) at $t=0$ [19]. A Josephson oscillation is then initiated by displacing the harmonic potential along the axial direction through a distance of $16 \mu \mathrm{m}$. Soon after this displacement, due to the onset of collapse there is no stationary solution to the GP equation. Numerically, the nonexistence of a stationary solution to the GP equation and the subsequent nonequilibrium dynamics of the condensate manifests in the destruction (delocalization) of the localized stationary wave function at $t=0$ and the generation of a nonequilibrium wave function spread over the entire spatial domain of discretization as seen in Fig. 5(b) at $t=16 \mathrm{~ms}$.

When there is a free Josephson tunneling, the condensate oscillates as a whole without significant modification of shape or local density. However, when there is a disruption
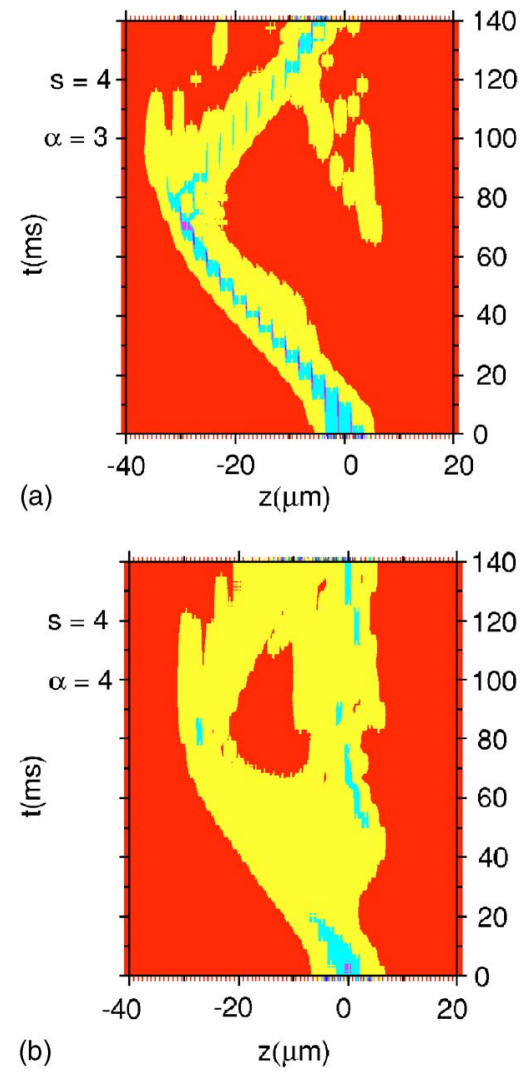

FIG. 4. (Color online) Contour plot of the one-dimensional probability density $P(z, t)$ of the BEC with $s=4$ and $n=-0.1$ when the harmonic potential is suddenly displaced along the axial direction through a distance $16 \mu \mathrm{m}$ at $t=0$ for a laser wavelength parameter $\alpha=$ (a) 3 and (b) 4 . The disruption of the Josephson oscillation has already started for $\alpha=3$ and is complete for $\alpha=4$.

of the Josephson tunneling and the BEC is given additional potential energy to oscillate by displacing the harmonic potential, the condensate is locally squeezed to a smaller region in space resulting in a very large density and thus inducing a collapse of the condensate as illustrated in Fig. 5.

The investigations of Refs. $[7,8]$ also provides answer to some of the questions raised in the present paper, e.g., how does the Josephson oscillation depend on the nonlinearity parameter $n$ and the optical-lattice potential strength $s$. Using a hydrodynamical model, the authors of Ref. [7] show for an elongated or cigar-shaped confinement $(\nu \ll 1)$ that the frequency of oscillation $\omega_{z} \equiv \nu \omega$ in a pure harmonic potential gets changed in the presence of the optical-lattice potential according to $\omega_{\mathrm{opt}}=\omega_{z} \sqrt{\mathrm{m} / \mathrm{m}^{*}}$, where $m$ is the atomic mass and $m^{*}$ is an effective mass. A good estimate of $m / m^{*}$ can be made by neglecting both the magnetic trapping and the atomatom interaction from the spectrum of the linear Schrödinger equation for the one-dimensional periodic optical-lattice potential. In this approximation $\mathrm{m} / \mathrm{m}^{*}$ is a universal function of the parameter $s$, calculated in Ref. [7]. This dependence of $\mathrm{m} / \mathrm{m}^{*}$ on $s$ has been used to calculate the dependence of Josephson frequency on $s$ [7] as quoted in Fig. 3 of this paper. The quantity $\mathrm{m} / \mathrm{m}^{*}$ also depends on nonlinearity $n$ and harmonic oscillator frequency $\omega$. However, the dependence of $m / m^{*}$ on $n$ and $\omega$ is weak for small values of these pa- 

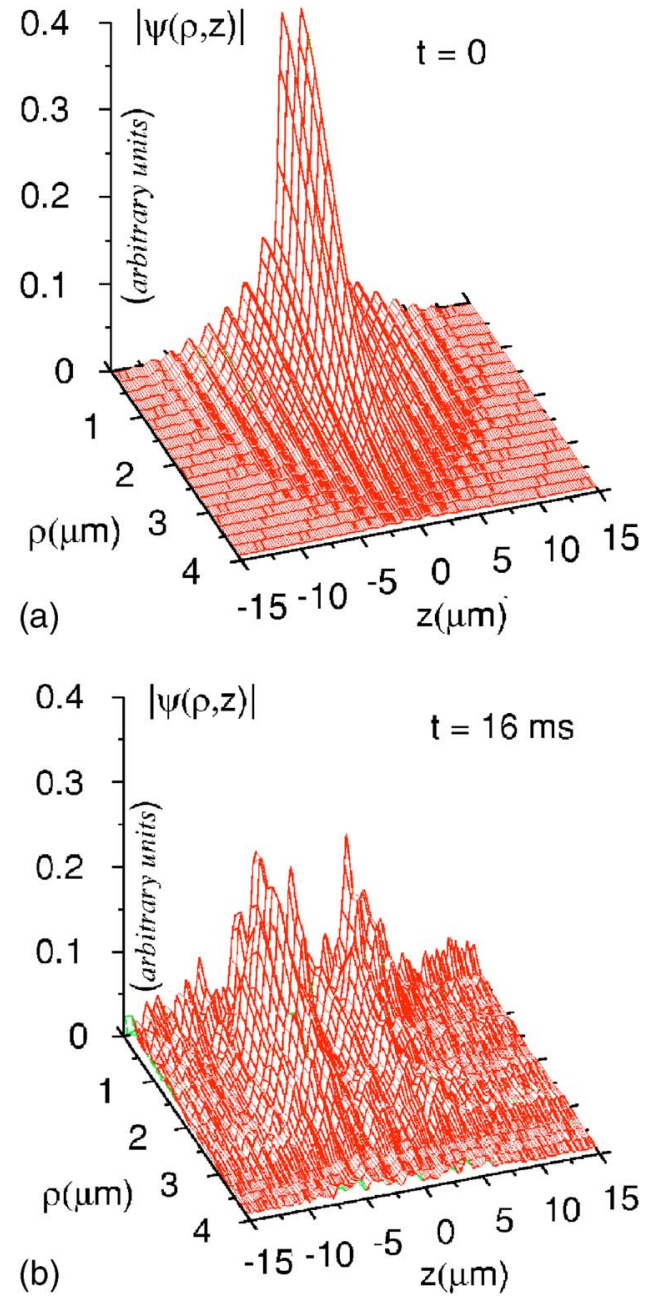

FIG. 5. (Color online) (a) The profile of the wave function $|\psi(\rho, y)|$ formed in the joint optical-lattice and harmonic potentials for $s=4, \alpha=3$, and $n=-0.5$ at $t=0$. (b) The profile of the wave function of (a) at $t=16 \mu \mathrm{m}$ after an axial displacement of the harmonic potential through $16 \mu \mathrm{m}$ at $t=0$.

rameters [8]. In Ref. [8] the authors study the dependence of $\mathrm{m} / \mathrm{m}^{*}$ on nonlinearity $n$ in a model and demonstrate the weak dependence for small and medium values of $n$. Consequently, the independence of the Josephson frequency on the nonlinearity parameter $n$ as well as the dependence of this frequency on the parameter $s$ as noted in Fig. 3 seems to be consistent with Refs. [7,8]. However, it should be noted that the studies of Refs. $[7,8]$ did not consider the Josephson oscillation for an attractive interaction and the possibility of collapse as in this paper. Hence, the conclusions of Refs. $[7,8]$ are only valid for repulsive atomic interaction and the present study extends them to the case of attractive atomic interactions. Moreover, the present study is based on the three-dimensional mean-field microscopic GP equations, whereas those of Refs. $[7,8]$ are based on hydrodynamical and one-dimensional mean-field models of the condensate.

\section{SUMMARY AND CONCLUSION}

We performed a numerical simulation based on an axially symmetric mean-field GP equation to address different aspects of the Josephson oscillation of an attractive BEC in a combined harmonic and periodic optical-lattice potentials. The latter potential is created by a standing-wave laser beam. We find that the Josephson frequency is virtually independent of the number of atoms in the condensate and the nature of atomic interaction: attractive or repulsive. The mean-field results for the Josephson frequencies for different opticallattice strengths are in qualitative agreement with experiment $[1]$ and other calculations $[1,6,7]$. However, there is some quantitative disagreement between different results. The Josephson frequency decreases with increasing optical-lattice strength $s$. As $s$ increases from 2 to 8 the frequency reduces from 8.5 to $4.5 \mathrm{~Hz}$. The dependence of the Josephson frequency on the laser wavelength is much weaker than its dependence on $s$. The Josephson frequency remains in the range $7.1 \pm 0.3 \mathrm{~Hz}$ for a variation of laser wave length in the region $1600 \mathrm{~nm}>\lambda>600 \mathrm{~nm}$. However, as $\lambda$ is increased past $2000 \mathrm{~nm}$ there is a disruption of the Josephson oscillation. For an attractive BEC with nonlinearity stronger than a critical value this disruption of Josephson oscillation could induce a collapse. These features of the Josephson oscillation could be tested experimentally and will provide a test for the mean-field model.

\section{ACKNOWLEDGMENT}

The work was supported in part by the CNPq of Brazil.
[1] F. S. Cataliotti, S. Burger, C. Fort, P. Maddaloni, F. Minardi, A. Trombettoni, A. Smerzi, and M. Inguscio, Science 293, 843 (2001).

[2] S. V. Pereverzev, S. Backaus, A. Loshak, J. C. Davis, and R. E. Packard, Nature (London) 388, 449 (1997).

[3] F. Dalfovo, S. Giorgini, L. P. Pitaevskii, and S. Stringari, Rev. Mod. Phys. 71, 463 (1999).

[4] P. Pedri, L. Pitaevskii, S. Stringari, C. Fort, S. Burger, F. S. Cataliotti, P. Maddaloni, F. Minardi, and M. Inguscio, Phys. Rev. Lett. 87, 220401 (2001).

[5] S. Burger, F. S. Cataliotti, C. Fort, F. Minardi, M. Inguscio, M.
L. Chiofalo, and M. P. Tosi, Phys. Rev. Lett. 86, 4447 (2001). [6] S. K. Adhikari, Eur. Phys. J. D 25, 161 (2003).

[7] M. Krämer, L. Pitaevskii, and S. Stringari, Phys. Rev. Lett. 88, 180404 (2002).

[8] M. Krämer, C. Menotti, L. Pitaevskii, and S. Stringari, Eur. Phys. J. D 27, 247 (2003).

[9] E. Sakellari, N. P. Proukakis, M. Leadbeater, and C. S. Adams, New J. Phys. 6, 42 (2004);C. Menotti, A. Smerzi, and A. Trombettoni, ibid. 5, 112 (2003);J. Links and H.-Q. Zhou, Lett. Math. Phys. 60, 275 (2002);S. Zhang and F. Wang, Phys. Lett. A 279, 231 (2001);L.-B. Fu, J. Liu, and S.-G. Chen, ibid. 
298, 388 (2002);I. Zapata, F. Sols, and A. J. Leggett, Phys. Rev. A 67, 021603(R) (2003).

[10] E. A. Donley, N. R. Claussen, S. L. Cornish, J. L. Roberts, E. A. Cornell, and C. E. Wieman, Nature (London) 412, 295 (2001).

[11] S. K. Adhikari, Phys. Rev. A 71, 053603 (2005); 66, 043601 (2002); 66, 013611 (2002);H. Saito and M. Ueda, ibid. 65, 033624 (2002);L. Santos and G. V. Shlyapnikov, ibid. 66, 011602(R) (2002);C. M. Savage, N. P. Robins, and J. J. Hope, ibid. 67, 014304 (2003);R. A. Duine and H. T. C. Stoof, ibid. 68, 013602 (2003);E. A. Calzetta and B. L. Hu, ibid. 68, 043625 (2003);W. Bao, D. Jaksch, and P. A. Markowich, J. Phys. B 37, 329 (2004);E. Sakellari, N. P. Proukakis, C. S. Adams, ibid. 37, 3681 (2004).

[12] S. Inouye, M. R. Andrews, J. Stenger, H. J. Miesner, D. M. Stamper-Kurn, and W. Katerlee, Nature (London) 392, 151 (1998);J. L. Roberts, J. P. Burke, N. R. Claussen, S. L. Cornish, E. A. Donley, and C. E. Wieman, Phys. Rev. A 64, 024702 (2001).
[13] J. Garnier, F. K. Abdullaev, and B. B. Baizakov, Phys. Rev. A 69, 053607 (2004).

[14] M. Greiner, O. Mandel, T. Esslinger, T. W. Hänsch, and I. Bloch, Nature (London) 415, 39 (2002).

[15] O. Morsch, J. H. Müller, M. Cristiani, D. Ciampini, and E. Arimondo, Phys. Rev. Lett. 87, 140402 (2001).

[16] H. M. J. M. Boesten, C. C. Tsai, B. J. Verhaar, and D. J. Heinzen, Phys. Rev. Lett. 77, 5194 (1996).

[17] S. K. Adhikari and P. Muruganandam, J. Phys. B 35, 2831 (2002); 36, 409 (2003).

[18] S. K. Adhikari, Phys. Rev. E 65, 016703 (2002).

[19] S. K. Adhikari, J. Phys. B 36, 2943 (2003).

[20] A. Smerzi, A. Trombettoni, P. G. Kevrekidis, and A. R. Bishop, Phys. Rev. Lett. 89, 170402 (2002);F. S. Cataliotti, L. Fallani, F. Ferlaino, C. Fort, P. Maddaloni, and M. Inguscio, New J. Phys. 5, 71 (2003).

[21] S. K. Adhikari, Phys. Lett. A 313, 211 (2003); 308, 302 (2003); J. Phys. B, 36, 2725 (2003). 